\title{
Predicting cognitive decline with non-clinical markers in Parkinson's disease (PRECODE-2)
}

\author{
Tayyabah Yousaf $^{1} \cdot$ Gennaro Pagano $^{1} \cdot$ Flavia Niccolini ${ }^{1} \cdot$ Marios Politis $^{1}$
}

Received: 14 September 2018 / Revised: 5 February 2019 / Accepted: 16 February 2019 / Published online: 28 February 2019

(c) The Author(s) 2019

\begin{abstract}
Objectives To investigate whether baseline $\left[{ }^{123}\right.$ I]FP-CIT SPECT and CSF markers can predict cognitive impairment (CI) in PD patients, and provide a profile of those most at risk.

Methods 262 de novo PD patients from the Parkinson's Progression Markers Initiative database were stratified into two CI groups at the 36-month follow-up: MoCA-defined diagnosis: PD patients who had a MoCA score <26; neuropsychological test-defined diagnosis: PD patients with MoCA-defined diagnosis and at least two test scores (of six; irrespective of test domain) greater than 1.5 standard deviation below the mean score in healthy controls. Predictive variables of CI were divided into deciles, providing us with ideal cutoff values for each variable.

Results At the 36-month follow-up, 108/262 (41.2\%) PD patients had CI as defined by the MoCA, of which 40/108 (37.0\%) had neuropsychological test-defined CI. Baseline CSF A $\beta 42$ (hazard ratio [HR]: 0.996, confidence interval [CI]: 0.992-0.999, $p=0.025)$, CSF total tau ([HR]: $1.023,[\mathrm{CI}]: 1.002-1.044, p=0.031)$ and caudate $\left[{ }^{123} \mathrm{I}\right] \mathrm{FP}-\mathrm{CIT}$ SPECT uptake ([HR]: 0.332, [CI]: $0.115-0.960, p=0.042)$ were predictors of CI. Patients with reduced CSF A $\beta 42(<384.6 \mathrm{pg} / \mathrm{mL})$, increased CSF total tau $(>45.0 \mathrm{pg} / \mathrm{mL})$ and reduced caudate $\left[{ }^{123} \mathrm{I}\right]$ FP-CIT SPECT uptake $(<1.82)$ had a $65 \%$ risk of developing CI at $36-$ month follow-up.

Conclusion We report a characteristic profile (reduced CSF A $\beta 42$, increased CSF total tau and reduced caudate [ ${ }^{123}$ I]FP-CIT SPECT uptake) that enables identification of early PD patients at risk of developing CI. These findings confirm previous reports of low CSF A $\beta 42$, elevated CSF total tau and reduced dopaminergic integrity being associated with cognitive decline in PD.
\end{abstract}

Keywords Parkinson's disease $\cdot$ Cognitive decline $\cdot$ Cognitive impairment $\cdot$ Predictors $\cdot$ CSF $\cdot\left[{ }^{123} \mathrm{I}\right] \mathrm{FP}-\mathrm{CIT}$ SPECT

\section{Introduction}

Cognitive impairment (CI) is an increasingly recognised complication of Parkinson's disease (PD), with significant impact including increased caregiver burden and mortality [1]. Mild cognitive impairment (MCI), in particular, is recognised as a distinct entity, and a probable prodromal state to PD dementia [1]. Up to $80 \%$ of Parkinson's disease patients develop dementia during the course of the disease,

Marios Politis

marios.politis@kcl.ac.uk

http://nig-politis.com/

1 Neurodegeneration Imaging Group, Institute of Psychiatry, Psychology and Neuroscience (IoPPN), Maurice Wohl Clinical Neuroscience Institute, King's College London, 125 Coldharbour Lane, Camberwell, London SE5 9NU, UK presenting with heterogeneous cognitive profiles and underlying disease pathology [1]. It is crucial to identify $\mathrm{CI}$ at an early stage to determine cognitive prognosis, given that $\mathrm{MCI}$ is a key risk factor for dementia [15].

Discovering meaningful end points that measure progression is one of the greatest challenges in PD therapeutics. The implementation of suboptimal methods in refining prognosis, diagnosis and tracking disease progression continues to hamper patient management and, particularly, clinical research [27]. Thus, validated biomarkers with a high degree of sensitivity and specificity represent an unmet need in PD, especially for the development of disease-modifying therapies.

Previous reports have demonstrated that vascular and Alzheimer-type (amyloid- $\beta$ plaques and tau neurofibrillary tangles) pathologies co-exist in PD, and may contribute to the development of CI in PD [16]. In PD, the degenerative 
process commences years before any initial signs of $\mathrm{CI}$, and cerebrospinal fluid (CSF) markers could identify these pathological changes. This is because the CSF has more physical contact with the brain compared to other fluids, as it is not separated from the brain by the tightly regulated blood-brain barrier. As a result, proteins or peptides that may be directly reflective of disease pathology or brain-specific activities are likely to diffuse into the CSF, therefore highlighting that the CSF is potentially the most informative fluid in biomarkers discovery for neurodegenerative disease prognosis [5]. Previous studies have reported that reduced amyloid- $\beta 42$ (A $\beta 42$ ) [28] and increased total-tau and phosphorylated-tau [8] levels in CSF are associated with cognitive decline in PD.

Several PET studies have also reported that dysfunction of the dopaminergic system also influences cognition in PD, with frontostriatal dopaminergic deficits affecting executive function and cholinergic deficits affecting memory and visuospatial function [19]. Striatal dopaminergic function has also been implicated in cognitive performance in PD, with Jokinen and colleagues reporting a positive correlation between caudate $\left[{ }^{18} \mathrm{~F}\right] \mathrm{DOPA}$ uptake and performance in visual and verbal memory [17] and Rinnie and colleagues reporting an association between reduced caudate $\left[{ }^{18} \mathrm{~F}\right]$ DOPA uptake and impairment in verbal fluency, working memory and attentional functioning [25].

These observations prompted the present study, which sought to determine whether the combined evaluation of different non-clinical markers would be more useful to identify PD patients most at risk of developing CI, as identifying a combination of imaging and CSF biomarkers of cognitive decline provides a fruitful and more accurate approach for diagnosis and disease progression, compared with using either modality alone.

In this study, we hypothesised that molecular markers of PD pathology may have value for predicting cognitive decline and we sought to investigate this by using longitudinal clinical data from the Parkinson's Progression Markers Initiative (PPMI) and baseline [ $\left.{ }^{123} \mathrm{I}\right] \mathrm{FP}-\mathrm{CIT}$ single photon emission computed tomography (SPECT) imaging and CSF molecular measures in a group of patients, who at baseline, had early de novo PD.

\section{Materials and methods}

\section{Participants and clinical characteristics}

Data were acquired from the PPMI database (http://www. ppmi-info.org/data), which is a 5-year observational, international, multi-centre prospective study that provides an insight into disease aetiology by collecting biannual neurological and cognitive evaluations and baseline CSF sampling
[22]. Of a total of 412 PD patients, we identified and included $262 \mathrm{PD}$ patients who were not cognitively impaired at baseline (MoCA $\geq 26$ ), had $\left[{ }^{123} \mathrm{I}\right] \mathrm{FP}-\mathrm{CIT}$ SPECT and CSF data, and a complete 36-month follow-up.

All PD patients underwent an initial screening visit followed by a baseline visit where demographic, CSF and $\left[{ }^{123} \mathrm{I}\right]$ FP-CIT SPECT measures were collected (Table 1). Motor, non-motor, cognitive and neuropsychological assessments were also collected at baseline. The follow-up period was either terminated at the time point when patients had developed CI $(n=108)$ or at the complete 36-month follow-up visit if they had not developed CI $(n=154)$.

Institutional review boards approved the study and written informed consent was obtained from all participants. All PD patients were recruited between 2010 and 2015, had a disease duration of less than 2 years prior to a screening visit, were not taking dopamine replacement therapy and presented with at least two of the following symptoms: bradykinesia, rigidity, and resting tremor, OR the presence of either an asymmetric resting tremor or asymmetric bradykinesia. PD diagnosis was confirmed by presence of dopamine transporter deficit on the $\left[{ }^{123} \mathrm{I}\right] \mathrm{FP}-\mathrm{CIT}$ dopamine transporter imaging.

\section{$\left[{ }^{123}\right.$ I]FP-CIT SPECT molecular imaging}

SPECT images were obtained $4 \pm 0.5 \mathrm{~h}$ after administrating an injection of approximately $185 \mathrm{MBq}\left[{ }^{123} \mathrm{I}\right] \mathrm{FP}-\mathrm{CIT}$. $\left[{ }^{123} \mathrm{I}\right]$ FP-CIT SPECT scans were analysed following the imaging technical operations manual (http://ppmi-info.org/). In brief, SPECT image volumes were spatially normalised to an Ioflupane template. The eight most prominent axial slices containing the striatum were summed and a standardised volume of interest (VOI) template was then applied to this image. VOI analyses were performed on the right and left caudate and putamen, employing the occipital region as the reference tissue. Specific binding ratios were calculated as the ratio of the putamen or caudate VOI count density divided by the occipital cortex count density minus one. This measure approximates the binding potential, $\mathrm{BP}_{\mathrm{ND}}$, when the radioligand is in equilibrium at the target site and has previously been reported with Ioflupane SPECT.

\section{CSF and plasma molecular markers}

CSF and plasma samples were collected at baseline from all participants enrolled in the study including CSF A $\beta 42$, CSF alpha-synuclein, CSF total-tau, CSF phosphorylated tau-181, serum insulin-like growth factor 1 (IGF-1), amyloid precursor protein (APP), apolipoprotein A1, EGF ELISA, urate and glucose. Additional information on how CSF 
Table 1 Demographic characteristics and CSF, plasma and imaging measures for PD patients who developed MoCA-defined cognitive impairment at the 3-year follow-up

\begin{tabular}{|c|c|c|c|}
\hline & All PD patients $(n=262)$ & $\begin{array}{l}\text { Cognitive impairment } \\
(n=108)\end{array}$ & $\begin{array}{l}\text { No cognitive } \\
\text { impairment } \\
(n=154)\end{array}$ \\
\hline Age at screening, mean $\pm \mathrm{SD}^{\mathrm{a}}$ & $61.08 \pm 9.47$ & $64.56 \pm 8.46$ & $58.64 \pm 9.41 *$ \\
\hline Gender, male, $\%(n)^{\mathrm{b}}$ & $63.7(167)$ & $73.1(79)$ & $57.1(88)^{*}$ \\
\hline Age at diagnosis, mean $\pm \mathrm{SD}^{\mathrm{a}}$ & $60.52 \pm 9.45$ & $63.97 \pm 8.54$ & $58.10 \pm 9.33^{*}$ \\
\hline $\mathrm{PD}$ duration (months), mean $\pm \mathrm{SD}^{\mathrm{b}}$ & $6.68 \pm 6.87$ & $7.06 \pm 7.11$ & $6.40 \pm 6.70$ \\
\hline Family history of $\mathrm{PD}, \%(n)^{\mathrm{b}}$ & $25.6(67)$ & $24.1(26)$ & $26.6(41)$ \\
\hline Year of education, mean $\pm \mathrm{SD}^{\mathrm{b}}$ & $15.73 \pm 2.97$ & $15.61 \pm 3.42$ & $15.81 \pm 2.62$ \\
\hline CSF A $\beta 1-42$ levels $\mathrm{pg} / \mathrm{mL}$, mean $\pm \mathrm{SD}^{\mathrm{b}}$ & $369.89 \pm 93.38$ & $350.04 \pm 90.07$ & $383.81 \pm 93.43^{*}$ \\
\hline $\mathrm{CSF}$ alpha-synuclein $\mathrm{pg} / \mathrm{mL}$, mean $\pm \mathrm{SD}^{\mathrm{b}}$ & $1860.22 \pm 774.80$ & $1808.73 \pm 715.97$ & $1896.33 \pm 813.87$ \\
\hline CSF phosphorylated tau- $181 \mathrm{pg} / \mathrm{mL}$, mean $\pm \mathrm{SD}^{\mathrm{b}}$ & $15.53 \pm 10.37$ & $15.06 \pm 11.33$ & $15.87 \pm 9.66$ \\
\hline CSF total tau $\mathrm{pg} / \mathrm{mL}$, mean $\pm \mathrm{SD}^{\mathrm{b}}$ & $44.06 \pm 17.14$ & $45.58 \pm 19.26$ & $42.99 \pm 15.45$ \\
\hline Apolipoprotein $\mathrm{A} 1 \mathrm{mg} / \mathrm{dL}$, mean $\pm \mathrm{SD}^{\mathrm{b}}$ & $166.98 \pm 34.87$ & $167.11 \pm 34.38$ & $166.89 \pm 35.51$ \\
\hline Amyloid precursor protein, mean $\pm \mathrm{SD}^{\mathrm{b}}$ & $43.50 \pm 2.64$ & $43.68 \pm 2.43$ & $43.37 \pm 2.82$ \\
\hline Glucose $\mathrm{mmol} / \mathrm{L}$, mean $\pm \mathrm{SD}^{\mathrm{b}}$ & $5.48 \pm 0.78$ & $5.54 \pm 0.85$ & $5.44 \pm 0.73$ \\
\hline Serum IGF-1 ng/mL, mean \pm SD $^{b}$ & $138.92 \pm 54.08$ & $137.24 \pm 56.29$ & $140.10 \pm 52.63$ \\
\hline Uric acid umol/L, mean $\pm \mathrm{SD}^{\mathrm{b}}$ & $316.26 \pm 77.52$ & $334.41 \pm 78.78$ & $303.53 \pm 74.26^{*}$ \\
\hline Caudate $\left[{ }^{123} \mathrm{I}\right] \mathrm{FP}-\mathrm{CIT}$, mean $\pm \mathrm{SD}^{\mathrm{a}}$ & $1.96 \pm 0.54$ & $1.92 \pm 0.59$ & $1.99 \pm 0.49$ \\
\hline Putamen $\left[{ }^{123} \mathrm{I}\right] \mathrm{FP}-\mathrm{CIT}$, mean $\pm \mathrm{SD}^{\mathrm{b}}$ & $0.82 \pm 0.27$ & $0.82 \pm 0.31$ & $0.83 \pm 0.25$ \\
\hline
\end{tabular}

${ }^{*} p$ values $<0.05\left({ }^{\mathrm{a}} t\right.$ test, ${ }^{\mathrm{b}}$ Mann-Whitney $U$ test $)$

samples were collected and analysed has been previously reportedly [18].

\section{Primary end point}

Cognitive decline was the primary outcome of the study, which was reviewed and established by the study physicians during follow-up visits. The follow-up period was either terminated at the time-point when patients had developed CI or at the end of the study period if they had not developed CI. Individuals were classified as having normal cognition or cognitive impairment as outlined in the PPMI protocol. Cognitive decline was evaluated using the Montreal Cognitive Assessment (MoCA), which is a scale for assessing global cognitive abilities. Additionally, a detailed cognitive battery, as previously described [31], was administered: the Hopkins Verbal Learning Test-Revised for memory; the Benton Judgement of Line Orientation 15-item (split-half) version for visuospatial function; the Letter-Number Sequencing for executive function and working memory; the Symbol-Digit Modalities Test for processing speed-attention; and semantic (animals, fruits and vegetables) fluency test. Cognitive decline was defined in two ways: 1) MoCA-defined diagnosis included all PD patients who had a MoCA score $<26$; 2) neuropsychological test-defined diagnosis included all
PD patients with MoCA-defined diagnosis, who also stated having cognitive decline and had at least two test scores (of HVLT Total Recall, HVLT Recognition Discrimination, Benton Judgement of Line Orientation, Letter Number Sequencing, Semantic Fluency Test and/or Symbol Digit Modalities; irrespective of test domain) greater than 1.5 standard deviation below the age and education-standardised mean score based on published standards in healthy controls, and no functional impairment due to cognitive impairment [31].

\section{Statistical analysis}

Statistical analyses were performed using Statistical Package for the Social Sciences (SPSS), version 22. For all variables, variance homogeneity and Gaussianity were tested using the Kolmogorov-Smirnov test. Continuous variables were expressed as mean \pm standard deviation, with betweengroup comparisons performed by $t$ test or Mann-Whitney $U$ test for normally or non-normally distributed variables, respectively. Categorical variables were expressed as proportion and compared using a $\chi^{2}$ test. Predictors of cognitive decline were identified using multivariate backward elimination regression, which included demographic, CSF, plasma 
and imaging measures. Only the time to occurrence of the first event was used in the backward elimination regression model. In order to determine a characteristic profile for patients most at risk of developing CI, patients were stratified into groups based on the biomarkers that were significant predictors of CI, as defined by the neuropsychological tests. Kaplan-Meier estimates and curves were generated, and comparisons were made using the log-rank (MantelCox) test. Statistical significance was set at $p<0.05$.

Table 2 Backward elimination regression analysis for cognitive decline as defined by the MoCA and neuropsychological tests

\begin{tabular}{lccccc}
\hline & HR & \multicolumn{2}{l}{$95.0 \%$ CI for } & $p$ value & Wald \\
\cline { 3 - 4 } & & HR & & \\
\cline { 3 - 4 } & & Lower & Upper & & \\
\hline MoCA-defined CI & & & & & \\
CSF A $\beta 1-42$ levels pg/mL & 0.998 & 0.996 & 1.000 & 0.092 & 2.840 \\
CSF total tau pg/mL & 1.009 & 0.995 & 1.023 & 0.217 & 1.522 \\
Caudate [123 I]FP-CIT & 0.945 & 0.538 & 1.660 & 0.843 & 0.039 \\
Age at screening & 1.048 & 1.024 & 1.073 & $<0.001$ & 15.377 \\
Gender & 1.318 & 0.791 & 2.195 & 0.289 & 1.123 \\
Neuropsychological test-defined CI & & & \\
CSF A $\beta 1-42$ levels pg/mL & 0.996 & 0.992 & 0.999 & 0.025 & 5.035 \\
CSF total tau pg/mL & 1.023 & 1.002 & 1.044 & 0.031 & 4.680 \\
Caudate [ ${ }^{123}$ I]FP-CIT & 0.332 & 0.115 & 0.960 & 0.042 & 4.146 \\
Age at screening & 1.076 & 1.028 & 1.126 & 0.001 & 10.084 \\
Gender & 1.479 & 0.626 & 3.490 & 0.372 & 0.797 \\
\hline
\end{tabular}

\section{Results}

\section{Characteristics of PD patients}

We studied $262 \mathrm{PD}$ patients who had a mean disease duration of $6.68 \pm 6.87$ months (Table 1 ). At the 36-month followup, 108/262 (41.2\%) PD patients had MoCA-defined CI, of which 40/108 (37.0\%) had neuropsychological test-defined CI. Patients who were cognitively impaired as defined by the MoCA were significantly older at baseline and at PD onset and had reduced CSF A $\beta 42(p<0.05$; Table 1$)$.

\section{Predictors of cognitive decline}

Backward elimination regression analysis for cognitive impairment defined by MoCA did not reveal any predictors of CI (Table 2). Backward elimination regression analysis for cognitive impairment defined by the neuropsychological tests revealed the following predictors, after adjusting for age and gender: CSF A 442 (hazard ratio [HR]: 0.996, Wald: 5.035, confidence interval [CI]: 0.992-0.999, $p=0.025$ ), CSF total tau ([HR]: 1.023, Wald: 4.680, [CI]: 1.002-1.044, $p=0.031)$ and caudate $\left[{ }^{123} \mathrm{I}\right] \mathrm{FP}-\mathrm{CIT}$ uptake ([HR]: 0.332, Wald: 4.146, [CI]: 0.115-0.960, $p=0.042$; Table 2).

Patients were then divided into subgroups according to: CSF A $\beta 42$, CSF total tau and caudate $\left[{ }^{123} \mathrm{I}\right]$ FP-CIT uptake. We divided these variables into deciles and identified the deciles with the highest power of predication, providing us with ideal cutoff values for each variable. We found that
Fig. 1 PD sub-phenotypes at varying risk of CI development. PD patients were grouped by CSF A $\beta 42$ (cutoff $384.6 \mathrm{pg}$ / $\mathrm{mL}$ ), CSF total tau (cutoff $45 \mathrm{pg} / \mathrm{mL})$ and caudate $\left[{ }^{123} \mathrm{I}\right] \mathrm{FP}-$ CIT uptake (cutoff 1.82). Green boxes indicate normal variables and red boxes indicate abnormal boxes. The percentages of CI development (yellow boxes) are shown at the end of the study (36-month follow-up)

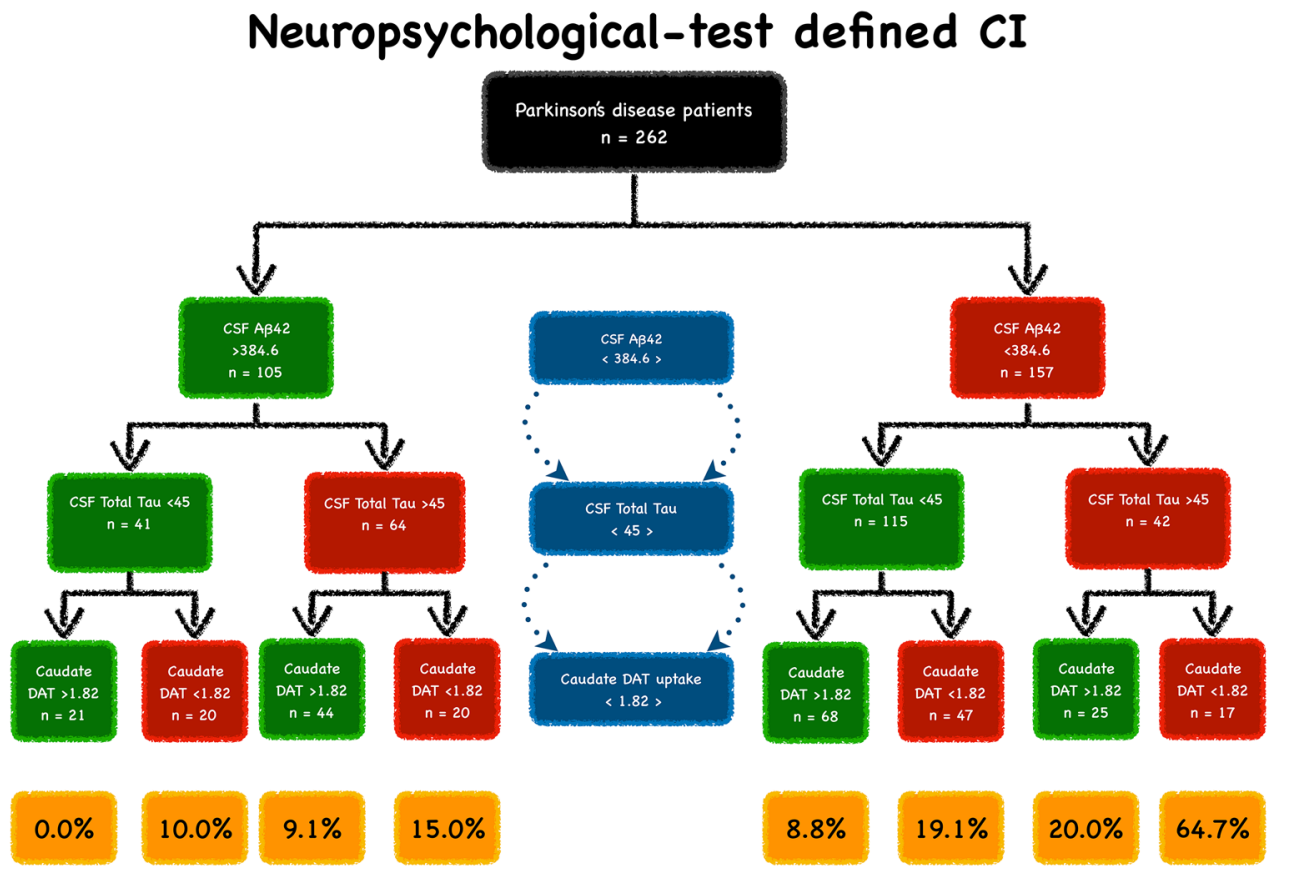


the best cutoff for CSF A $\beta 42$ was $384.6 \mathrm{pg} / \mathrm{mL}, 45 \mathrm{pg} / \mathrm{mL}$ for CSF total tau and 1.82 for caudate [ $\left.{ }^{123} \mathrm{I}\right] \mathrm{FP}-\mathrm{CIT}$ uptake.

PD patients with CSF A $\beta 42<384.6 \mathrm{pg} / \mathrm{mL}$, CSF total tau $>45.0 \mathrm{pg} / \mathrm{mL}$ and caudate $\left[{ }^{123} \mathrm{I}\right] \mathrm{FP}-\mathrm{CIT}$ uptake $<1.82$ had a $65 \%$ risk of developing CI, as defined by the neuropsychological tests, at a 36-month follow-up (Fig. 1). Patients with normal biomarkers did not develop CI. Patients with only one abnormal biomarker had an $8.8-10.0 \%$ risk of developing CI over a period of 36 months. Patients who had a combination of two abnormal markers had 15.0-20.0\% risk of CI development over a period of 36 months (Fig. 2).

\section{Discussion}

This pilot study demonstrated that a combined evaluation of biological and neuroimaging markers enables the identification of $65 \%$ of drug-naïve PD patients that will develop CI over a 36-month follow-up. CSF A $\beta 42$, CSF total tau and caudate $\left[{ }^{123}\right.$ I]FP-CIT uptake are predictors of CI. According to these measures, PD patients can be categorised into eight subgroups — each with a different conversion rate to CI depending exclusively on how many abnormal measures they have (Fig. 1). Patients with reduced CSF A $\beta 42$, increased CSF total tau and reduced caudate $\left[{ }^{123}\right.$ I] FP-CIT uptake have a $65 \%$ conversion to $\mathrm{CI}$, whereas patients who only have one abnormal measure have between 8.8 and $10.0 \%$ conversion rate to $\mathrm{CI}$. These findings confirm previous reports of low CSF A $\beta 42$, elevated CSF total tau and reduced dopaminergic integrity being associated with cognitive decline in PD [8, $17,25,28]$.

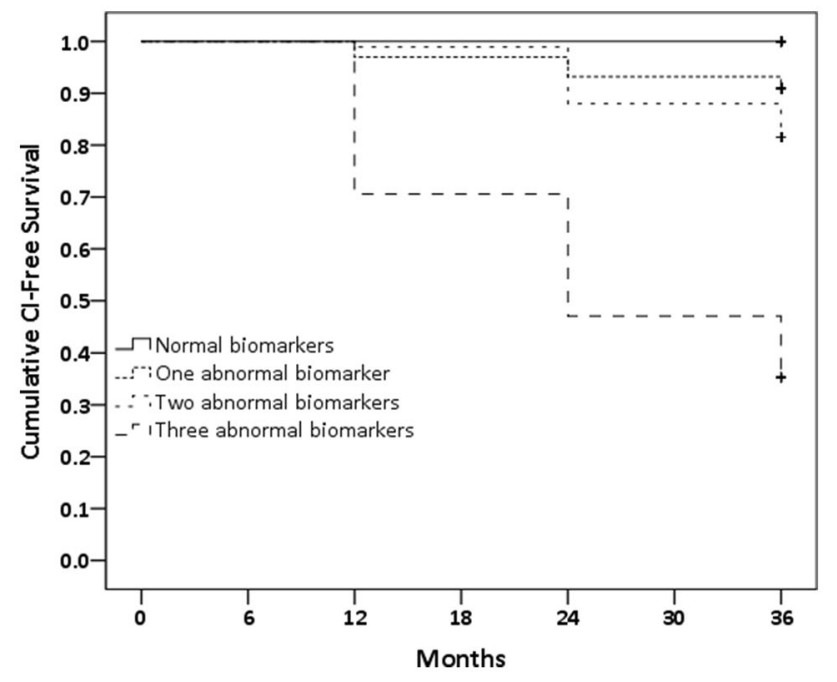

Fig. 2 Kaplan-Meier overall survival curves for the development of CI for each subgroup. Log Rank (Mantel-Cox) $=53.03 p<0.0001$. CI cognitive impairment
Although we did not find any predictors for MoCAdefined CI, this may be due to the fact that these particular biomarkers were not sufficient to detect a change in MoCA scores over a 36-month period. Furthermore, neuropsychological test-defined CI was done by a subjective complaint of cognitive decline by the patient at the visit follow-up and via a neuropsychological battery, which was selected to identify cognitive deficits typically occurring in PD, independent of motor abilities. This, therefore, suggests that the biomarker profile reflects subtle, accurate cognitive changes in $\mathrm{PD}$, as opposed to a loss of four points on the MoCA.

We propose that the current findings provide a simple characteristic profile of PD patients at risk of CI, which could not only be utilised in clinical settings to identify and correctly monitor PD patients who are most at risk of developing CI, but offer the opportunity to inform treatment decisions and bring personalised medicine into clinical practice. When taken as single markers, CSF A $\beta 42$, CSF total tau and caudate $\left[{ }^{123}\right.$ I]FP-CIT uptake are inadequate to identify PD patients at higher risk of cognitive decline, but taken together, these measures are able to predict cognitive decline in $65 \%$ of the cases.

Our findings are in line with the Norwegian ParkWest study, which reported a relationship between reduced CSF A $\beta 42$ levels and cognitive deficits in the memory domain [3], as well as lower CSF A $\beta 42$ levels predicting the development of dementia in PD [4]. Other studies that enrolled more advanced $\mathrm{PD}$ patients also concluded that $\mathrm{PD}$ patients exhibiting low CSF A $\beta 42$ levels at baseline have an increased susceptibility of developing CI [9]. Previous, smaller longitudinal studies demonstrated that caudate DAT binding can predict cognitive decline [23] and higher CSF total-tau and phospho-tau have an association with recall, naming, recognition and visuoperceptive neuropsychological deficits [8]. Our findings also corroborate with the study conducted by Caspell-Garcia and colleagues [7], who similarly assessed predictive biomarkers of cognitive decline in de novo PD patients from PPMI. Caspell-Garcia et al. [7] reported that reduced striatal DAT availability and low CSF A $\beta 42$ levels predicted cognitive decline, alongside diffuse cortical thinning and two SNPs: the COMT val158met and $B D N F$ val66met, though increased CSF total tau was not found to be a predictor of cognitive decline. The novelty of our study is that it was designed to identify cutoffs for the predictive biomarkers, which could potentially be employed in the clinical setting. Once validated in other cohorts, these cutoffs provide a clinically-relevant profile that can be employed in clinical trials.

By revealing a characteristic profile of PD patients most at risk of developing CI, it may propel development of mechanism-specific interventions through the identification of suitable subgroups for clinical trials and provision of objective measures of disease suppression. Objective, 
quantifiable characteristics of pathological processes are particularly crucial for future disease-modifying therapy trials for patients at high risk of cognitive decline. The high attrition rate for drugs in clinical development is predominantly due to the inability of the industry to predict a drug candidate's performance early, and with a large degree of certainty. Biomarkers hold the potential to substantially accelerate product development and reduce drug development costs by predicting drug efficacy and discriminating between suitable or unsuitable candidates for a given drug. Success with new therapies would rapidly lead to the widespread application of biomarkers in the clinical management of cognitive disorders in PD. Here, we have provided a combination of biomarkers which, taken together, have the ability to predict $\mathrm{CI}$ in $65 \%$ of early PD cases. This predictive, characteristic profile could tackle the challenges attached to clinical trials by enabling the correct identification of appropriate candidates for trials aiming to prevent or delay cognitive decline in PD.

We show that $\left[{ }^{123} \mathrm{I}\right] \mathrm{FP}-\mathrm{CIT}$ deficit in the caudate is vital for CI development. Caudate dopaminergic function has been recognised as a fundamental node in supporting cognitive domains. In people exposed to the neurotoxin 1-methyl4-phenyl-1,2,3,6 tetrahydropyridine (MPTP), which causes a selective dopaminergic lesion, deficits in visuospatial and executive functions were noted [29]. Patients that had a stroke of the caudate showed a deterioration in their intellectual function within 2 years of the event [6]. Previous findings have also illustrated that loss of dopaminergic projections to the caudate correlate with degree of dementia in PD [26], alongside others reporting that a reduction in caudate uptake of $\left[{ }^{18} \mathrm{~F}\right] \mathrm{DOPA}$ is associated with verbal and visual memory functioning, verbal fluency and, delayed recall [25], highlighting that the functional integrity of presynaptic dopaminergic synthesis is crucial, not only for motor, but also for cognitive function in PD. Although the putamen has inherited the motor functionality classically ascribed to the basal ganglia, studies have demonstrated a role for the putamen in learning and memory [30]. We did not find an association between $\left[{ }^{123} \mathrm{I}\right] \mathrm{FP}-\mathrm{CIT}$ putamen binding and cognitive impairment, which is in line with findings from $[14,24]$, who did not find a correlation between putamen uptake of $\left[{ }^{18} \mathrm{~F}\right]$ fluorodopa and deficits in neuropsychiatric assessments. This highlights that correct identification of PD patients at risk of CI should include assessment of $\left[{ }^{123} \mathrm{I}\right]$ FP-CIT uptake in the caudate.

Although inclusions containing $\alpha$-synuclein is the hallmark pathology of PD, PD is now recognised as a more complex clinicopathological entity, with multiple coexisting processes. Neuropathological studies have highlighted the co-occurrence of $\alpha$-synuclein inclusions and amyloid plaques in neocortical regions in PD patients. Studies have reported that PD patients with dementia have increased $\beta$-amyloid using Pittsburgh Compound B PET imaging and a positive correlation between AD pathology and severity of PD with dementia [13].

A $\beta 42$, in particular, is believed to potentiate $\alpha$-synuclein aggregation and accumulation of $A \beta 42$ and tau is exacerbated by $\alpha$-synuclein. Furthermore, A $\beta 42$ can contribute to the development of Lewy body disease through promoting $\alpha$-synuclein aggregation, supporting the role of AD pathology in the pathogenesis of cognitive decline [2]. Reduced CSF A $\beta 42$, however, likely reflects the exacerbated accumulation of $A \beta 42$ in the brain. Preclinical studies have suggested that plaques act as an A $\beta 42$ "sink", therefore preventing soluble $A \beta 42$ from being transported from the brain to the CSF [10]. Fagan was the first study to investigate this relationship in humans, revealing that low CSF A $\beta 142$ reflect higher levels of $A \beta 42$ deposition in the cortex of $A D$ patients, supporting the hypothesis [11]. No data, however, has been showed in PD.

The microtubule-associated protein tau gene (MAPT) has been consistently associated with PD risk, with studies reporting cross-sectional reductions in CSF total tau in PD patients compared to controls [21]. Increased CSF total tau has been suggested to reflect neuronal, preferentially axonal, damage or degeneration, therefore inducing cognitive impairment. Although studies have reported the independent predictive nature of CSF A $\beta 142$ [4], Compta and colleagues reported high levels of CSF tau in PD patients with dementia, compared to non-demented PD patients [8]. Recently, Gomperts and colleagues imaged tau in patients with Lewy body diseases using a highly selective radioligand: $\left[{ }^{18} \mathrm{~F}\right] \mathrm{AV}-1451$ [12]. They reported that patients with Lewy body dementia and cognitively impaired PD patients had increased $\left[{ }^{18} \mathrm{~F}\right] \mathrm{AV}-1451$ binding in the inferior temporal gyrus and precuneus, which was associated with increased cognitive impairment.

The mechanisms underlying early cognitive impairment in PD have been demonstrated to be multifactorial. In addition to caudate dopaminergic dysfunction and comorbid $\mathrm{AD}$ pathology, the underlying pathology of cognitive impairment in PD in also believed to include non-dopaminergic neurotransmitter dysfunction (serotonin, norepinephrine and acetylcholine), cortical/limbic Lewy body pathology and cerebrovascular damage, as well as cortical atrophy and genetic factors. However, PD patient with low caudate $\left[{ }^{123} \mathrm{I}\right]$ FP-CIT uptake, low CSF A $\beta 142$ and elevated CSF total tau may represent a distinct subset of subjects at higher risk of cognitive decline. Lashley and colleagues put forward the notion that subgroups of PD patients could be differentiated based on significant quantitative differences in their cortical $\mathrm{A} \beta$ burden, which is in turn associated with $\alpha$-synuclein load in cerebral cortex [20].

The major limitation of this study is related to the grouping. This method is relatively stringent in terms of 
inclusion characteristics. Although this is beneficial for controlling the variables hypothesised to be predictive of cognitive decline, it has led to a restricted number of patients within each cohort. Furthermore, given the lack of adjustment for multiple testing, it is important to note that these results are highly exploratory, and therefore require validation from larger studies. It is also imperative to highlight that the cognitive battery was limited, whereby there was a lack of coverage of certain domains such as language abilities, and an uneven coverage of other domains. Although this level of assessment provided a lower diagnostic certainty than a more extensive neuropsychological evaluation, the cognitive tests included are easy to administer and freely available. Furthermore, classification of CI was done according to a predefined criterion that has been employed across several studies exploring $\mathrm{CI}$ in PD [7, 31]. Furthermore, although at the time of follow-up, the patients were classified as having developed cognitive impairment, we were not able to account for the possibility of patients reverting back to a cognitively normal condition after 36 months, which may have been due to initiation/ change of treatment or mood fluctuations.

CSF is an accessible source of brain-derived proteins, which reflect molecular changes in the brain and $\left[{ }^{123} \mathrm{I}\right] \mathrm{FP}-$ CIT SPECT provides valuable information on brain structure and function in the neuropsychiatric aspects of PD. Reduction of $\left[{ }^{123} \mathrm{I}\right] \mathrm{FP}-\mathrm{CIT}$ uptake within the putamen is commonly used in clinical practice to assist the diagnosis of PD and here we demonstrated a potential clinical applicability of $\left[{ }^{123} \mathrm{I}\right] \mathrm{FP}-\mathrm{CIT}$ uptake within the caudate assisting with disease prognosis, specifically cognitive decline. We show that having low CSF A $\beta 42$, elevated CSF total tau, and low $\left[{ }^{123}\right.$ I] FP-CIT caudate binding increase the risk of cognitive decline in PD patients by up to $65 \%$. These measures, taken together, may also reflect a characteristic profile of early PD patients most at risk of developing $\mathrm{CI}$ over the course of the disease. However, given the restricted number of patients within each subgroup, further studies with larger cohorts of PD patients are required to confirm and validate these exploratory findings.

\begin{abstract}
Author contributions Miss TY: Study concept and design, statistical analysis and interpretation of data and drafting of the manuscript. Dr. GP: study concept and design, study supervision, interpretation of data and revision of the manuscript for important intellectual content. Dr. FN: critical revision of the manuscript for important intellectual content. Prof. MP: study concept and design, study supervision, critical revision of the manuscript for important intellectual content and final approval of the manuscript.
\end{abstract}

Funding Data used in the preparation of this article were obtained from the Parkinson's Progression Markers Initiative (PPMI) database (http:// www.ppmi-info.org/data). For up-to-date information on the study, visit http://www.ppmi-info.org. PPMI-a public-private partnership-is sponsored by the Michael J. Fox Foundation for Parkinson's Research
(MJFF) and is co-funded by MJFF, Abbvie, Avid Radiopharmaceuticals, Biogen Idec, Bristol-Myers Squibb, Covance, Eli Lilly \& Co., F. Hoffman-La Roche, Ltd., GE Healthcare, Genentech, GlaxoSmithKline, Lundbeck, Merck, MesoScale, Piramal, Pfizer and UCB. Industry partners are contributing to PPMI through financial and in-kind donations and are playing a lead role in providing feedback on study parameters through the Industry Scientific Advisory Board (ISAB). Through close interaction with the study, the ISAB is positioned to inform the selection and review of potential progression markers that could be used in clinical testing. Miss Yousaf, Dr. Pagano, Dr Niccolini and Prof. Politis report no disclosures.

\section{Compliance with ethical standards}

Conflicts of interest The authors declare no potential conflicts of interest with respect to the research, authorship and/or publication of this article.

Ethical standards This study been approved by the appropriate ethics committee and has therefore been performed in accordance with the ethical standards laid down in the 1964 Declaration of Helsinki and its later amendments. All persons gave informed consent proper to their inclusion in the study. Details that might disclose the identity of the subjects under study have been omitted.

Open Access This article is distributed under the terms of the Creative Commons Attribution 4.0 International License (http://creativeco mmons.org/licenses/by/4.0/), which permits unrestricted use, distribution, and reproduction in any medium, provided you give appropriate credit to the original author(s) and the source, provide a link to the Creative Commons license, and indicate if changes were made.

\section{References}

1. Aarsland D, Andersen K, Larsen JP, Lolk A, Kragh-Sorensen P (2003) Prevalence and characteristics of dementia in Parkinson disease: an 8-year prospective study. Arch Neurol 60:387-392

2. Aarsland D, Kurz MW (2010) The epidemiology of dementia associated with Parkinson disease. J Neurol Sci 289:18-22

3. Alves G, Bronnick K, Aarsland D, Blennow K, Zetterberg H, Ballard C, Kurz MW, Andreasson U, Tysnes OB, Larsen JP, Mulugeta E (2010) CSF amyloid-beta and tau proteins, and cognitive performance, in early and untreated Parkinson's disease: the Norwegian ParkWest study. J Neurol Neurosurg Psychiatry 81:1080-1086

4. Alves G, Lange J, Blennow K, Zetterberg H, Andreasson U, Forland MG, Tysnes OB, Larsen JP, Pedersen KF (2014) CSF Abeta42 predicts early-onset dementia in Parkinson disease. Neurology 82:1784-1790

5. Anoop A, Singh PK, Jacob RS, Maji SK (2010) CSF biomarkers for Alzheimer's disease diagnosis. Int J Alzheimers Dis 2010:606802. https://doi.org/10.4061/2010/606802

6. Bokura H, Robinson RG (1997) Long-term cognitive impairment associated with caudate stroke. Stroke 28:970-975

7. Caspell-Garcia C, Simuni T, Tosun-Turgut D, Wu IW, Zhang Y, Nalls M, Singleton A, Shaw LA, Kang JH, Trojanowski JQ, Siderowf A, Coffey C, Lasch S, Aarsland D, Burn D, Chahine LM, Espay AJ, Foster ED, Hawkins KA, Litvan I, Richard I, Weintraub D, Parkinson's Progression Markers I (2017) Multiple modality biomarker prediction of cognitive impairment in prospectively followed de novo Parkinson disease. PLoS One 12:e0175674 
8. Compta Y, Marti MJ, Ibarretxe-Bilbao N, Junque C, Valldeoriola F, Munoz E, Ezquerra M, Rios J, Tolosa E (2009) Cerebrospinal tau, phospho-tau, and beta-amyloid and neuropsychological functions in Parkinson's disease. Mov Disord 24:2203-2210

9. Compta Y, Pereira JB, Rios J, Ibarretxe-Bilbao N, Junque C, Bargallo N, Camara A, Buongiorno M, Fernandez M, Pont-Sunyer C, Marti MJ (2013) Combined dementia-risk biomarkers in Parkinson's disease: a prospective longitudinal study. Parkinsonism Relat Disord 19:717-724

10. DeMattos RB, Bales KR, Parsadanian M, O’Dell MA, Foss EM, Paul SM, Holtzman DM (2002) Plaque-associated disruption of CSF and plasma amyloid-beta (Abeta) equilibrium in a mouse model of Alzheimer's disease. J Neurochem 81:229-236

11. Fagan AM, Mintun MA, Mach RH, Lee SY, Dence CS, Shah AR, LaRossa GN, Spinner ML, Klunk WE, Mathis CA, DeKosky ST, Morris JC, Holtzman DM (2006) Inverse relation between in vivo amyloid imaging load and cerebrospinal fluid Abeta42 in humans. Ann Neurol 59:512-519

12. Gomperts SN, Locascio JJ, Makaretz SJ, Schultz A, Caso C, Vasdev N, Sperling R, Growdon JH, Dickerson BC, Johnson K (2016) Tau positron emission tomographic imaging in the Lewy body diseases. JAMA Neurol 73:1334-1341

13. Gomperts SN, Rentz DM, Moran E, Becker JA, Locascio JJ, Klunk WE, Mathis CA, Elmaleh DR, Shoup T, Fischman AJ, Hyman BT, Growdon JH, Johnson KA (2008) Imaging amyloid deposition in Lewy body diseases. Neurology 71:903-910

14. Holthoff-Detto VA, Kessler J, Herholz K, Bonner H, Pietrzyk U, Wurker M, Ghaemi M, Wienhard K, Wagner R, Heiss WD (1997) Functional effects of striatal dysfunction in Parkinson disease. Arch Neurol 54:145-150

15. Janvin CC, Larsen JP, Aarsland D, Hugdahl K (2006) Subtypes of mild cognitive impairment in Parkinson's disease: progression to dementia. Mov Disord 21:1343-1349

16. Jellinger KA, Attems J (2008) Prevalence and impact of vascular and Alzheimer pathologies in Lewy body disease. Acta Neuropathol 115:427-436

17. Jokinen P, Bruck A, Aalto S, Forsback S, Parkkola R, Rinne JO (2009) Impaired cognitive performance in Parkinson's disease is related to caudate dopaminergic hypofunction and hippocampal atrophy. Parkinsonism Relat Disord 15:88-93

18. Kang JH, Irwin DJ, Chen-Plotkin AS, Siderowf A, Caspell C, Coffey CS, Waligorska T, Taylor P, Pan S, Frasier M, Marek K, Kieburtz K, Jennings D, Simuni T, Tanner CM, Singleton A, Toga AW, Chowdhury S, Mollenhauer B, Trojanowski JQ, Shaw LM, Parkinson's Progression Markers I (2013) Association of cerebrospinal fluid beta-amyloid 1-42, T-tau, P-tau181, and alphasynuclein levels with clinical features of drug-naive patients with early Parkinson disease. JAMA Neurol 70:1277-1287 I )
19. Kehagia AA, Barker RA, Robbins TW (2010) Neuropsychological and clinical heterogeneity of cognitive impairment and dementia in patients with Parkinson's disease. Lancet Neurol 9:1200-1213

20. Lashley T, Holton JL, Gray E, Kirkham K, O’Sullivan SS, Hilbig A, Wood NW, Lees AJ, Revesz T (2008) Cortical alpha-synuclein load is associated with amyloid-beta plaque burden in a subset of Parkinson's disease patients. Acta Neuropathol 115:417-425

21. Montine TJ, Shi M, Quinn JF, Peskind ER, Craft S, Ginghina C, Chung KA, Kim H, Galasko DR, Jankovic J, Zabetian CP, Leverenz JB, Zhang J (2010) CSF Abeta(42) and tau in Parkinson's disease with cognitive impairment. Mov Disord 25:2682-2685

22. Parkinson Progression Marker I (2011) The Parkinson progression marker initiative (PPMI). Prog Neurobiol 95:629-635

23. Ravina B, Marek K, Eberly S, Oakes D, Kurlan R, Ascherio A, Beal F, Beck J, Flagg E, Galpern WR, Harman J, Lang AE, Schwarzschild M, Tanner C, Shoulson I (2012) Dopamine transporter imaging is associated with long-term outcomes in Parkinson's disease. Mov Disord 27:1392-1397

24. Rinne JO, Portin R, Ruottinen H, Nurmi E, Bergman J, Haaparanta M, Solin O (2000) Cognitive impairment and the brain dopaminergic system in Parkinson disease - [F-18]fluorodopa positron emission tomographic study. Arch Neurol Chic 57:470-475

25. Rinne JO, Portin R, Ruottinen H, Nurmi E, Bergman J, Haaparanta M, Solin O (2000) Cognitive impairment and the brain dopaminergic system in Parkinson disease: $[18 \mathrm{~F}]$ fluorodopa positron emission tomographic study. Arch Neurol 57:470-475

26. Rinne JO, Rummukainen J, Paljarvi L, Rinne UK (1989) Dementia in Parkinson's disease is related to neuronal loss in the medial substantia nigra. Ann Neurol 26:47-50

27. Shtilbans A, Henchcliffe C (2012) Biomarkers in Parkinson's disease: an update. Curr Opin Neurol 25:460-465

28. Skogseth RE, Bronnick K, Pereira JB, Mollenhauer B, Weintraub D, Fladby T, Aarsland D (2015) Associations between cerebrospinal fluid biomarkers and cognition in early untreated Parkinson's Disease. J Parkinsons Dis 5:783-792

29. Stern Y, Langston JW (1985) Intellectual changes in patients with MPTP-induced parkinsonism. Neurology 35:1506-1509

30. van Beilen M, Leenders KL (2006) Putamen FDOPA uptake and its relationship tot cognitive functioning in PD. J Neurol Sci 248:68-71

31. Weintraub D, Simuni T, Caspell-Garcia C, Coffey C, Lasch S, Siderowf A, Aarsland D, Barone P, Burn D, Chahine LM, Eberling J, Espay AJ, Foster ED, Leverenz JB, Litvan I, Richard I, Troyer MD, Hawkins KA, Parkinson's Progression Markers I (2015) Cognitive performance and neuropsychiatric symptoms in early, untreated Parkinson's disease. Mov Disord 30:919-927 